\title{
Valores de referencia personalizados para el índice de pulsatilidad medio de la arteria uterina durante el embarazo según paridad, medición transvaginal y presión arterial
}

\author{
Héctor Oviedo-Cruz, ${ }^{*}$ Erika R. Carrasco-Blancas ${ }^{1}$ y Marco A. Cortés-Martínez²
}

${ }^{1}$ Dirección General; ${ }^{2}$ Departamento de Bioquímica. Centro Médico para Atención Fetal Especializada, Ciudad de México, México

\begin{abstract}
Resumen
Introducción: Los rangos de referencia de población específica para el índice de pulsatilidad medio de la arteria uterina (IPmAUt) durante el embarazo han demostrado valor en el cuidado prenatal. Objetivo: Construir valores de referencia para el IPmAUt durante el embarazo, personalizados por características maternas, medición transvaginal y presión arterial en una población mexicana. Métodos: Estudio transversal de 2286 embarazos normales de feto único en la Ciudad de México. La presión arterial y el IPmAUt se midieron mediante metodología estandarizada. Se construyeron rangos de referencia por gestación. Los efectos de las variables independientes se probaron mediante regresión lineal múltiple. Resultados: La mediana del IPmAUt entre las 11 y 41 semanas disminuyó de 1.714 a 0.523. El percentil 95 disminuyó de 2.600 a 0.653. La paridad sin preeclampsia previa representó el principal efecto sobre el IPmAUt. La presión arterial media tuvo efecto sobre el IPmAUt por interacción con la paridad. La preeclampsia previa tuvo efecto sobre el IPmAUt por interacción con las características maternas. Se obtuvo un factor de corrección para medición transvaginal. Conclusiones: El IPmAUt disminuye normalmente según la placentación y adaptación materna al embarazo. Los efectos de la paridad sobre la presión arterial y el IPmAUt podrían reflejar remodelación cardiovascular posterior a la gestación.
\end{abstract}

PALABRAS CLAVE: Atención prenatal. Ultrasonografía Doppler. Presión arterial. Valores de referencia. Modelación específica para el paciente. México.

\section{Reference values for uterine artery mean pulsatility index throughout pregnancy customized by parity, transvaginal measurement and blood pressure}

\begin{abstract}
Introduction: Population-specific reference ranges for uterine artery (UtA) mean pulsatility index (PI) throughout pregnancy have been shown to be of value in antenatal care. Objective: To construct reference values for UtA mean PI throughout pregnancy, customized by maternal characteristics, transvaginal measurement and blood pressure in a Mexican population. Methods: Cross-sectional study in 2286 normal singleton pregnancies in Mexico City. Blood pressure and UtA mean PI were measured using standardized methodology. Reference ranges by gestation were constructed. The effects of independent variables were tested by multiple linear regression. Results: UtA mean PI median value between 11 and 41 weeks decreased from 1.714 to 0.523 . The $95^{\text {th }}$ percentile decreased from 2.600 to 0.653 . Previous parity without preeclampsia had the main effect on UtA mean PI. Mean blood pressure had an effect on UtA mean PI by interaction with parity. Previous preeclampsia had an effect on UtA mean PI by interaction with maternal characteristics. A correction factor was obtained for transvaginal
\end{abstract}

Correspondencia:

*Héctor Oviedo-Cruz

E-mail: HectorOC@ @emafe.org.mx
Fecha de recepción: 15-08-2021

Fecha de aceptación: 28-10-2021

DOI: 10.24875/GMM.21000502
Gac Med Mex. 2022;158:50-56

Disponible en PubMed

www.gacetamedicademexico.com

0016-3813/@ 2021 Academia Nacional de Medicina de México, A.C. Publicado por Permanyer. Este es un artículo open access bajo la licencia CC BY-NC-ND (http://creativecommons.org/licenses/by-nc-nd/4.0/). 
measurement. Conclusions: UtA mean PI usually decreases according to placentation and maternal adaptation to pregnancy. The effects of parity on blood pressure and UtA mean PI might reflect cardiovascular remodeling after gestation.

KEYWORDS: Prenatal care. Doppler ultrasonography. Blood pressure. Reference values. Patient-specific modeling. Mexico.

\section{Introducción}

El ultrasonido Doppler de la arteria uterina se ha convertido en un método rutinario para evaluar de manera no invasiva la circulación uteroplacentaria durante la gestación, para lo cual se han utilizando varios índices Doppler, ${ }^{1-3}$ entre los cuales se ha preferido el índice de pulsatilidad (IP) porque describe mejor la forma de la onda de velocidad, aunque son necesarias consideraciones estadísticas para una implementación adecuada. ${ }^{1,4-6}$

Aunque los rangos de referencia para el IP medio de la arteria uterina (IPmAUt) durante el embarazo ya han sido determinados, ${ }^{5,7}$ es de esperar variaciones en los valores normales debido a las diferencias de población, mejoras en el equipo y las guías metodológicas. ${ }^{7}$ Se ha demostrado que los rangos de referencia específicos para la población diana son valiosos en la predicción, el diagnóstico y el tratamiento de la preeclampsia y la restricción del crecimiento intrauterino, ${ }^{8-10}$ así como en la predicción de otros resultados perinatales adversos. ${ }^{11,12}$ Se han recomendado gráficos actualizados y específicos de la población para la toma de decisiones. ${ }^{7}$

En el primer trimestre del embarazo, la ecografía Doppler uterina se pueden realizar por vía transabdominal o transvaginal. ${ }^{1,5}$ Aunque las ondas de velocidad del flujo de la arteria uterina durante el embarazo son diferentes en las mediciones transabdominal y transvaginal, ${ }^{13}$ no se ha investigado ningún método de corrección.

Se ha propuesto la adición de variables a los modelos de referencia, como la paridad ${ }^{14} \mathrm{y}$ la presión arterial media (PAM), ${ }^{3}$ para mejorar el valor clínico de las mediciones Doppler. El historial médico y otras características maternas se han utilizado para estandarizar los valores Doppler del IP de la arteria uterina en múltiplos de la mediana (MoM), ${ }^{15}$ método que ha demostrado su valor en la detección de la

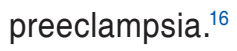

El objetivo de este estudio fue construir valores de referencia para el IPmAUt durante el embarazo en una población mexicana y personalizarlos por características maternas, medición transvaginal y presión arterial.

\section{Métodos}

Estudio transversal prospectivo de mujeres con embarazo de feto único, quienes acudieron a ecografía rutinaria entre las 11 a 41 semanas de gestación en un centro y laboratorio de medicina materno-fetal de la Ciudad de México. Los criterios de inclusión fueron cursar con embarazo espontáneo normal con un feto vivo normal en la fecha de la exploración y gozar de buena salud; los criterios de no inclusión fueron antecedentes médicos de hipertensión crónica, diabetes mellitus, lupus eritematoso sistémico, síndrome antifosfolípidos o cualquier otra enfermedad, independientemente de conllevar riesgo para enfermedad vascular. Se excluyeron los fetos con anomalías cromosómicas, estructurales o del crecimiento. Se incluyeron 2286 mujeres embarazadas, reclutadas de enero de 2017 a diciembre de 2018. La edad gestacional al momento de la ecografía tuvo una distribución multimodal debido a la agenda rutinaria; como tamaño de muestra se estableció un mínimo de 30 mediciones por cada grupo de gestación. Hubo 893 embarazos entre las 11 y 14 semanas de gestación, 351 entre las 15 y 18 semanas, 647 entre las 19 y 25 semanas, 96 entre las 26 y 29 semanas, 263 entre las 30 y 36 semanas y 36 entre las 37 y 41 semanas. El protocolo de investigación fue aprobado por el comité de ética local conforme con los estándares éticos de la Declaración de Helsinki de la Asociación Médica Mundial; todas los mujeres consintieron en participar en el estudio previa información del mismo. Se garantizó el anonimato de las participantes.

\section{Características de las pacientes}

Las características maternas registradas incluyeron edad materna, origen racial (hispano/caucásico, afrocaribeño, sur de Asia, este de Asia y otro/mixto), hábito de fumar durante el embarazo (fumadora, nunca o dejó de fumar), antecedentes familiares de preeclampsia en la madre o hermana de la paciente (sí o no) y antecedentes obstétricos, incluida la paridad (paridad o nulípara si no hubo embarazos previos de 24 semanas de 
gestación o más), y embarazo previo con preeclampsia (sí o no). Se midieron peso y talla maternos.

La edad gestacional se determinó mediante biometría fetal en el primer o segundo trimestre; el último periodo menstrual se usó para las visitas tardías, solo si hubo concordancia con el ultrasonido.

\section{Medición del índice Doppler}

Los exámenes Doppler de la arteria uterina se realizaron utilizando un equipo de ultrasonido Voluson 730 Expert, Voluson E6 o Voluson E10 (GE Healthcare Technologies $®)$, que cuenta con transductores transvaginal y transabdominal multifrecuencia. Los exámenes fueron realizados por dos observadores certificados (fetalmedicine.org) de acuerdo con un método estandarizado. ${ }^{5}$ Se examinaron transabdominalmente 2000 embarazos entre las semanas 11 y 41 , incluidos 607 entre las semanas 11 y 14, más 286 embarazos examinados entre las semanas 11 y 14 con una sonda transvaginal.

\section{Medición de la presión arterial}

Las mediciones de la presión arterial se obtuvieron utilizando dispositivos electrónicos automatizados compatibles con la FDA 510(k) (HEM-7121, Omron®, Japón), que se calibraron antes y a intervalos regulares durante el estudio. El procedimiento se realizó de acuerdo con un protocolo estandarizado modificado que se describe detalladamente en otro estudio. ${ }^{17}$

\section{Análisis estadístico}

Los valores de referencia para el IPmAUt se construyeron contra la edad gestacional, con base en el método estadístico descrito por Royston y Wright. ${ }^{18}$

Los efectos de las características maternas, la medición transvaginal y la presión arterial en el IP medio log (MoM) se probaron mediante regresión lineal múltiple. Se utilizó el análisis escalonado para detectar factores de confusión y efectos de la interacción. La bondad del ajuste se evaluó mediante índices de rendimiento y análisis de residuos. El modelo final para estimar la mediana del IP medio $\log (\mathrm{MoM})$ se eligió por equilibrio entre la bondad del ajuste, la coherencia clínica y la simplicidad del modelo. ${ }^{19,20}$

A continuación, se construyeron rangos de referencia multivariables del IPmAUt en el embarazo con base en el método descrito por Royston y Wright, ${ }^{18}$ modificado de la siguiente manera: los percentiles ajustados para el IPmAUt se calcularon a partir del modelo de mediana del IP medio log (MoM) por gestación por regresión lineal múltiple, y la desviación estándar estimada a partir de la desviación estándar de los residuos no escalados; se asumió consistencia si los residuos absolutos escalados no mostraron tendencia en todas las variables independientes incluidas en el modelo.

Se investigaron los supuestos estadísticos y se aplicaron pruebas paramétricas, no paramétricas o la transformación de variables según fuera necesario. También se probaron los supuestos de regresión lineal, transformando las variables conforme fuera necesario. El nivel de significación para todas las pruebas estadísticas fue $p<0.05$.

El conjunto de datos se creó a partir de las bases de datos y programas de cómputo Astraia ${ }^{\circledR}$ (Alemania) y Excel@ (EE. UU.) y estará disponible desde http:// doi.org/10.6084/m9.figshare.12991157. El análisis estadístico se realizó con los programas Excel y MATLAB® (EE. UU.).

\section{Resultados}

Las características maternas y la presión arterial de la población de estudio se muestran en la Tabla 1. Solo 33/2286 (1.4 \%) eran adolescentes y 18/2286 (0.8\%) tuvieron paridad de tres o más.

El mejor modelo para expresar la relación entre el IPmAUt y la edad gestacional requirió la transformación de la variable dependiente en su logaritmo natural. La mediana resultó un polinomio de tercer grado y la desviación estándar resultó una función lineal de la edad gestacional (Tabla 2).

La Figura 1 es un gráfico de dispersión de los valores del IPmAUt observados conforme a la edad gestacional, junto con la mediana estimada y los percentiles relevantes, también tabulados en la Tabla 3.

Todas las características maternas se incluyeron en el modelo multivariable final, excepto el tabaquismo ( $F=0.754, p=0.385$ ); los coeficientes y las interacciones se presentan en la Tabla 4 junto con la DE estimada. La historia obstétrica, definida como paridad con o sin preeclampsia previa, tuvo el principal efecto sobre el IP medio log (MoM) de la arteria uterina. El efecto más representativo de la presión arterial sobre el IP medio log (MoM) de la arteria uterina fue el de la PAM por interacción con la paridad, que se representa específicamente en la Figura 2. 
Tabla 1. Características maternas y presión arterial en la población de estudio $(n=2286)$

\begin{tabular}{|c|c|c|}
\hline Variable & Mediana & RIC \\
\hline Edad materna (años) & 33.0 & $30.0,36.0$ \\
\hline Talla materna $(\mathrm{cm})$ & 160.0 & $156.0,164.0$ \\
\hline Peso materno (kg) & 65.3 & $58.6,73.1$ \\
\hline \multirow[t]{2}{*}{ Índice de masa corporal } & 25.3 & $23.1,28.1$ \\
\hline & Media & DE \\
\hline \multirow[t]{2}{*}{$\begin{array}{l}\text { Presión arterial (mm Hg) } \\
\text { Sistólica } \\
\text { Diastólica } \\
\text { Presión arterial media }\end{array}$} & $\begin{array}{l}101.5 \\
66.3 \\
78.1\end{array}$ & $\begin{array}{l}9.4 \\
7.2 \\
7.4\end{array}$ \\
\hline & n & $\%$ \\
\hline $\begin{array}{l}\text { Etnia } \\
\text { Hispana/caucásica } \\
\text { Afrocaribeña } \\
\text { Sur de Asia } \\
\text { Este de Asia } \\
\text { Otra/mixta }\end{array}$ & $\begin{array}{c}1918 \\
4 \\
2 \\
36 \\
326\end{array}$ & $\begin{array}{c}83.9 \\
0.2 \\
0.1 \\
1.6 \\
14.3\end{array}$ \\
\hline $\begin{array}{l}\text { Paridad } \\
\text { Nulípara } \\
\text { Paridad sin preeclampsia previa } \\
\text { Paridad, preeclampsia previa }\end{array}$ & $\begin{array}{c}1245 \\
1014 \\
27\end{array}$ & $\begin{array}{c}54.5 \\
44.4 \\
1.2\end{array}$ \\
\hline Fumadora de cigarrillo & 13 & 0.6 \\
\hline Dejó de fumar & 297 & 13.0 \\
\hline Historia familiar de preeclampsia & 58 & 2.5 \\
\hline
\end{tabular}

Los modelos se codificaron en una hoja de cálculo de Excel, que estará disponible en http://doi. org/10.6084/m9.figshare.12991322

\section{Discusión}

Como se describió previamente en otra población, ${ }^{5}$ la edad gestacional fue el principal determinante del IPmAUt en nuestro estudio: se observó una disminución significativa desde el primer al segundo trimestre del embarazo, estabilizándose en el tercero, y una nueva disminución leve al término. La primera disminución brusca del IPmAUt puede estar relacionada con el proceso de conversión de las arterias espirales en arterias uteroplacentarias durante la placentación normal; la desorganización de las capas musculoelásticas de estos vasos, causada por la invasión citotrofoblástica, provoca una disminución dramática del tono vascular en la circulación uteroplacentaria. ${ }^{21}$ Después de eso, la caída menos pronunciada en el IPmAUt puede estar relacionada con una invasión trofoblástica más

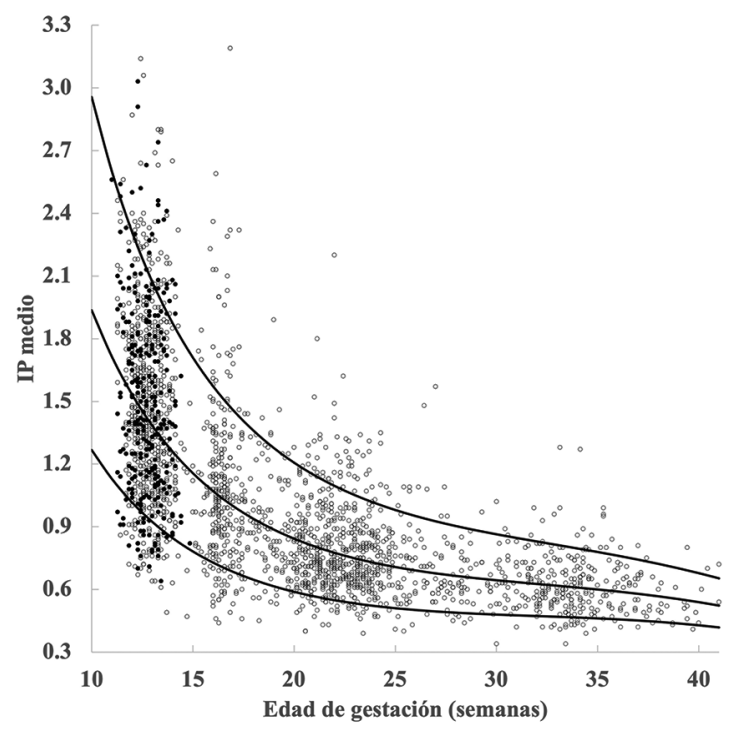

Figura 1. Valores de referencia del índice de pulsatilidad (IP) medio de la arteria uterina relacionado con la edad de gestacional en la población de estudio. Las mediciones se realizaron mediante ultrasonido transvaginal $(\bullet)$ y transabdominal $\left({ }^{\circ}\right)$. Se muestran la mediana estimada con los percentiles 5 y 95.

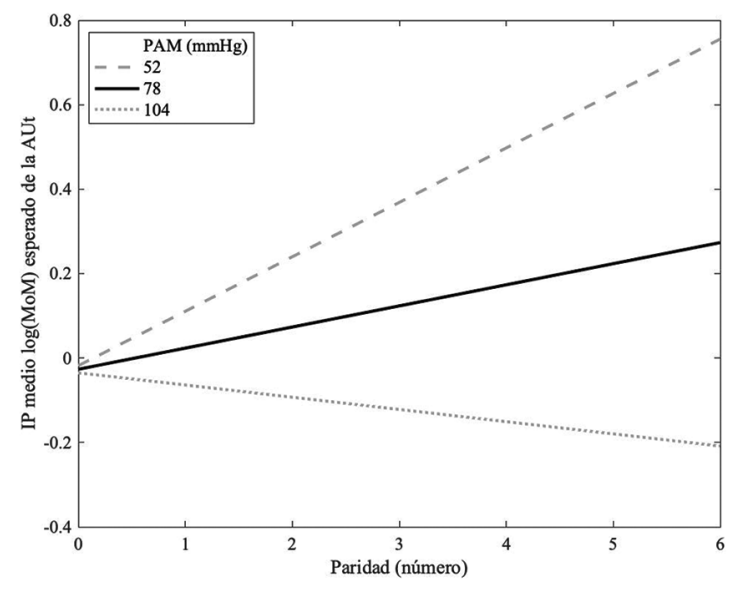

Figura 2. Interacción entre la presión arterial media (PAM, $\mathrm{mm} \mathrm{Hg}$ ) y la paridad (número) en el modelo multivariable del log (MoM) para el índice de pulsatilidad (IP) medio de la arteria uterina por gestación. Aut: arteria uterina; IP: índice de pulsatilidad; MoM: múltiplos de la mediana; PAM: presión arterial media;

lenta durante la última etapa del embarazo y con cambios maternos durante las últimas etapas del embarazo en el gasto cardiaco, el volumen sistólico, la resistencia vascular sistémica, el volumen sanguíneo y la viscosidad de la sangre. 22,23

En comparación con los valores del IPmAUt obtenidos en Barcelona con el mismo método estandarizado 
Tabla 2. Modelo del índice de pulsatilidad medio de arteria uterina por gestación

\begin{tabular}{|c|c|c|c|c|}
\hline Término & Estimado & IC $95 \%$ & $t$ & $p$ \\
\hline $\begin{array}{l}\text { Mediana* } \\
\text { Intersección } \\
E G \\
E G^{2} \\
E G^{3}\end{array}$ & $\begin{array}{c}2.52345 \\
-0.03622 \\
1.53 \times 10^{-4} \\
-2.28 \times 10^{-7}\end{array}$ & $\begin{array}{c}2.1499,2.8970 \\
-0.0439,-0.0285 \\
1.04 \times 10^{-4}, 2.03 \times 10^{-4} \\
-3.27 \times 10^{-7},-1.30 \times 10^{-7}\end{array}$ & $\begin{array}{c}13.2 \\
-9.2 \\
6.1 \\
-4.5\end{array}$ & $\begin{array}{l}<0.001 \\
<0.001 \\
<0.001 \\
<0.001\end{array}$ \\
\hline $\begin{array}{l}\text { Desviación estándar } \\
\text { Intersección } \\
\text { EG }\end{array}$ & $\begin{array}{c}0.2967 \\
-5.62 \times 10^{-4}\end{array}$ & $\begin{array}{c}0.2765,0.3169 \\
-7.00 \times 10^{-4},-4.23 \times 10^{-4}\end{array}$ & $\begin{array}{l}28.8 \\
-7.9\end{array}$ & $\begin{array}{l}<0.001 \\
<0.001\end{array}$ \\
\hline
\end{tabular}

Tabla 3. Valores de referencia del índice de pulsatilidad medio de la arteria uterina por gestación

\begin{tabular}{|c|c|c|c|}
\hline \multirow[t]{2}{*}{ EG (semanas) } & \multicolumn{3}{|c|}{ Índice de pulsatilidad medio } \\
\hline & p5 & Mediana & p95 \\
\hline 11 & 1.129 & 1.714 & 2.600 \\
\hline 12 & 1.016 & 1.532 & 2.310 \\
\hline 13 & 0.923 & 1.383 & 2.071 \\
\hline 14 & 0.846 & 1.259 & 1.874 \\
\hline 15 & 0.782 & 1.156 & 1.710 \\
\hline 16 & 0.729 & 1.070 & 1.573 \\
\hline 17 & 0.684 & 0.998 & 1.457 \\
\hline 18 & 0.647 & 0.938 & 1.360 \\
\hline 19 & 0.615 & 0.886 & 1.277 \\
\hline 20 & 0.589 & 0.843 & 1.207 \\
\hline 21 & 0.567 & 0.806 & 1.147 \\
\hline 22 & 0.549 & 0.775 & 1.096 \\
\hline 23 & 0.533 & 0.749 & 1.052 \\
\hline 24 & 0.521 & 0.727 & 1.014 \\
\hline 25 & 0.510 & 0.707 & 0.980 \\
\hline 26 & 0.502 & 0.691 & 0.952 \\
\hline 27 & 0.495 & 0.677 & 0.926 \\
\hline 28 & 0.489 & 0.665 & 0.904 \\
\hline 29 & 0.484 & 0.654 & 0.883 \\
\hline 30 & 0.480 & 0.644 & 0.864 \\
\hline 31 & 0.476 & 0.635 & 0.847 \\
\hline 32 & 0.473 & 0.626 & 0.830 \\
\hline 33 & 0.469 & 0.618 & 0.813 \\
\hline 34 & 0.466 & 0.609 & 0.797 \\
\hline 35 & 0.462 & 0.600 & 0.779 \\
\hline 36 & 0.457 & 0.590 & 0.762 \\
\hline 37 & 0.452 & 0.579 & 0.743 \\
\hline 38 & 0.445 & 0.567 & 0.723 \\
\hline 39 & 0.438 & 0.554 & 0.701 \\
\hline 40 & 0.429 & 0.539 & 0.678 \\
\hline 41 & 0.418 & 0.523 & 0.653 \\
\hline
\end{tabular}

de medición, ${ }^{5}$ los rangos de referencia de nuestro estudio resultaron progresivamente más bajos a lo largo del descenso desde el primer al segundo trimestre del embarazo, e incluso más bajos hacia el descenso al término de la gestación. Esta diferencia progresiva en el flujo uteroplacentario a medida que avanza la gestación puede, hipotéticamente, reflejar un efecto poblacional sobre la adaptación materna durante el embarazo.

Aunque la heterogeneidad metodológica ${ }^{24}$ puede explicar las diferencias entre nuestro estudio y otro, ${ }^{7}$ en este último se combinaron los datos de dos poblaciones diferentes. Como encontramos diferencias étnicas entre nuestra muestra de población, similar a otros investigadores, ${ }^{15}$ concluimos que la etnia debe ser considerada para construir rangos de referencia para índices de la arteria uterina. Una contribución de nuestra investigación es la interacción entre las características étnicas y otras características maternas después de la corrección por gestación; hemos planteado la hipótesis de que las diferencias étnicas en el flujo uteroplacentario a lo largo de la gestación están relacionadas con la expresión fenotípica de diversos rasgos poligénicos/multifactoriales, que actúan de forma epigenética sobre la placentación y la adaptación materna al embarazo. Esta hipótesis es congruente con patrones diferenciales de expresión de ARN en embarazos normales en comparación con embarazos patológi$\cos ,{ }^{25}$ por lo que se justifica más investigación sobre este tema.

En nuestro análisis, el efecto conocido de la PAM sobre el IPmAUt ${ }^{3}$ resultó secundario a la paridad, que a su vez constituyó el efecto principal. Esta interacción entre PAM e historia obstétrica en los resultados de la ecografía Doppler materna es congruente con lo que se conoce respecto al efecto de la paridad sobre la presión arterial y las mediciones de la ecografia Doppler de la arteria uterina durante el embarazo ${ }^{26}$ y cómo estos índices se asocian a la 
Tabla 4: Modelo multivariable del índice de pulsatilidad medio log (MoM) de la arteria uterina

\begin{tabular}{|c|c|c|c|c|}
\hline Término & Estimado & IC 95\% & $\mathrm{t}$ & $p$ \\
\hline $\begin{array}{l}\text { Valor medio esperado* } \\
\text { Intersección } \\
\text { Edad materna }(\mathrm{años}) \\
\text { Talla materna }(\mathrm{cm}) \\
\text { Peso materno }(\mathrm{kg}) \\
\text { PAM (mm Hg) } \\
\text { Medición transvaginal }\end{array}$ & $\begin{array}{r}2.6317 \\
-0.0711 \\
-0.0159 \\
-0.0010 \\
-0.0003 \\
0.2932\end{array}$ & $\begin{array}{r}0.5693,4.6942 \\
-0.1334,-0.0088 \\
-0.0289,-0.0030 \\
-0.0026,0.0006 \\
-0.0025,0.0018 \\
0.0987,0.4876\end{array}$ & $\begin{array}{r}2.5 \\
-2.2 \\
-2.4 \\
-1.2 \\
-0.3 \\
3.0\end{array}$ & $\begin{array}{l}0.012 \\
0.025 \\
0.016 \\
0.212 \\
0.768 \\
0.003\end{array}$ \\
\hline $\begin{array}{l}\text { Etnia }^{\dagger} \\
\text { Este de Asia } \\
\text { Otra/mixta }\end{array}$ & $\begin{array}{r}0.0363 \\
-0.0040\end{array}$ & $\begin{array}{l}-0.7445,0.8171 \\
-0.0393,0.0313\end{array}$ & $\begin{array}{r}0.1 \\
-0.2\end{array}$ & $\begin{array}{l}0.927 \\
0.826\end{array}$ \\
\hline $\begin{array}{l}\text { Paridad }^{\dagger} \\
\text { Número } \\
\text { Paridad, no PE previa } \\
\text { Paridad, PE previa } \\
\text { Dejó de fumar } \\
\text { Historia familiar de PE }\end{array}$ & $\begin{array}{r}0.2793 \\
-0.3326 \\
0.1242 \\
0.3421 \\
-0.0566\end{array}$ & $\begin{array}{r}0.0838,0.4747 \\
-0.4841,-0.1811 \\
-1.1136,1.3620 \\
0.0875,0.5967 \\
-0.1297,0.0166\end{array}$ & $\begin{array}{r}2.8 \\
-4.3 \\
0.2 \\
2.6 \\
-1.5\end{array}$ & $\begin{array}{r}0.005 \\
<0.001 \\
0.844 \\
0.008 \\
0.130\end{array}$ \\
\hline $\begin{array}{l}\text { Interacciones } \\
\text { Edad: Talla } \\
\text { Edad: Este de Asia } \\
\text { Edad: PE previa } \\
\text { Edad: dejó de fumar } \\
\text { Peso: transvaginal } \\
\text { Peso: Este del Asia } \\
\text { Peso: no PE previa } \\
\text { Peso: PE previa } \\
\text { PAM: paridad número } \\
\text { Otro, mixto: dejó de fumar } \\
\text { Paridad número: dejó de fumar } \\
\text { Historia familiar de PE: PE previa } \\
\text { Desviación estándar }\end{array}$ & $\begin{array}{r}0.0004 \\
0.0229 \\
-0.0324 \\
-0.0109 \\
-0.0044 \\
-0.0156 \\
0.0042 \\
0.0108 \\
-0.0030 \\
0.1013 \\
0.0664 \\
1.3104 \\
0.2734\end{array}$ & $\begin{array}{r}0.0001,0.0008 \\
0.0012,0.0446 \\
-0.0571,-0.0077 \\
-0.0187,-0.0032 \\
-0.0073,-0.0015 \\
-0.0257,-0.0055 \\
0.0020,0.0063 \\
0.0019,0.0196 \\
-0.0055,-0.0006 \\
0.0081,0.1946 \\
0.0092,0.1236 \\
0.7441,1.8768\end{array}$ & $\begin{array}{r}2.2 \\
2.1 \\
-2.6 \\
-2.8 \\
-2.9 \\
-3.0 \\
3.8 \\
2.4 \\
-2.5 \\
2.1 \\
2.3 \\
4.5\end{array}$ & $\begin{array}{r}0.025 \\
0.038 \\
0.010 \\
0.006 \\
0.003 \\
0.002 \\
<0.001 \\
0.017 \\
0.014 \\
0.033 \\
0.023 \\
<0.001\end{array}$ \\
\hline
\end{tabular}

función cardiovascular materna, ${ }^{27}$ según la remodelación cardiovascular posterior a la gestación. ${ }^{28} \mathrm{La}$ contribución especial de nuestro estudio es la posibilidad de evaluar individualmente este efecto de interacción. A partir de nuestro modelo, planteamos la hipótesis de que la paridad sin preeclampsia ejerce un efecto favorable sobre la remodelación cardiovascular, dependiendo de la composición corporal; por el contrario, la preeclampsia previa tiene un efecto perjudicial sobre la remodelación cardiovascular en función de la predisposición familiar, la composición corporal y el envejecimiento cardiovascular.

Aunque el número es pequeño, la falta de efecto del tabaquismo en nuestro modelo puede explicarse hipotéticamente por alguna reacción de la placenta, por sobreproducción de factor de crecimiento placentario, como lo describen Llurba et al.:29 sin embargo, este problema está más allá del alcance de nuestro estudio.
Encontramos un factor de corrección estadísticamente significativo para la medición transvaginal del IPmAUt, congruente con las diferencias conocidas de las ondas de velocidad de flujo de la arteria uterina en el embarazo entre la medición transabdominal y transvaginal; ${ }^{13}$ en nuestros datos, este efecto también se ajustó por el peso materno. Esta contribución práctica de nuestro modelo aún no se ha probado desde el punto de vista clínico.

\section{Conclusiones}

El IPmAUt disminuye durante el embarazo normal según la placentación y la adaptación materna al embarazo. Las diferencias étnicas en el flujo uteroplacentario a lo largo de la gestación pueden estar relacionadas con rasgos poligénicos/multifactoriales. Los efectos de la paridad sobre la presión arterial y el IPmAUt pueden reflejar la remodelación 
cardiovascular después de la gestación. Un factor de corrección para la medición transvaginal fue estadísticamente significativo. Se necesitan más investigaciones para demostrar el valor real de esta herramienta clínica en la predicción, el diagnóstico y el tratamiento de la preeclampsia, la restricción del crecimiento intrauterino y los nuevos grupos emergentes de alto riesgo, como las mujeres embarazadas con infecciones por las diferentes variantes de SARS-CoV-2.

\section{Financiamiento}

La presente investigación no recibió ninguna beca específica de agencias de los sectores públicos, comercial, o sin ánimo de lucro.

\section{Conflicto de intereses}

Los autores declaran no tener conflicto de intereses.

\section{Responsabilidades éticas}

Protección de personas y animales. Los autores declaran que para esta investigación no se realizaron experimentos en seres humanos ni en animales.

Confidencialidad de los datos. Los autores declaran que siguieron los protocolos de su centro de trabajo sobre la publicación de datos de pacientes.

Derecho a la privacidad y consentimiento infor-

mado. Los autores declaran que en este artículo no aparecen datos de pacientes.

\section{Bibliografía}

1. Kaminopetros $\mathrm{P}$, Higueras MT, Nicolaides $\mathrm{KH}$. Doppler study of uterine artery blood flow: comparison of findings in the first and second trimesters of pregnancy. Fetal Diagn Ther. 1991;6:58-64.

2. Hernández-Andrade E, Brodszki J, Lingman G, Gudmundsson S, Molin J Maršál K. Uterine artery score and perinatal outcome. Ultrasound Obstet Gynecol. 2002;19:438-442.

3. Flo K, Wilsgaard T, Acharya G. A new non-invasive method for measuring uterine vascular resistance and its relationship to uterine artery Doppler indices: a longitudinal study. Ultrasound Obstet Gynecol. 2011;37:538-542.

4. Thompson MO, Vines KS, Aqualina J, Harrington KF. Uterine artery Doppler blood flow resistance indices in the second half of pregnancy: measures of central tendency, distribution, inter-ratio dependency, and explanation of variation. Ultrasound Obstet Gynecol. 2001;18:55.

5. Gómez O, Figueras F, Fernández S, Bennasar M, Martínez JM, Puerto B, et al. Reference ranges for uterine artery mean pulsatility index at 11 41 weeks of gestation. Ultrasound Obstet Gynecol. 2008;32:128-132.

6. Timor-Tritsch IE, Monteagudo A. Scanning techniques in obstetrics and gynaecology. En: Wladimiroff JW, Eik-Nes SH, editores. Ultrasound in obstetrics and gynaecology. Escocia: Elsevier; 2009.
7. Medina CN, Figueroa DH, Guzmán-Huerta M, Hernández-Andrade E. Valores de referencia del índice de pulsatilidad de las arterias uterina y umbilical durante el embarazo. Ginecol Obstet Mex. 2006;74:509-515.

8. Harrington K, Goldfrad C, Carpenter RG, Campbell S. Transvaginal uterine and umbilical artery Doppler examination of 12-16 weeks and the subsequent development of pre-eclampsia and intrauterine growth retardation. Ultrasound Obstet Gynecol. 1997;9:94-100.

9. Martin AM, Bindra R, Curcio P, Cicero S, Nicolaides KH. Screening for pre-eclampsia and fetal growth restriction by uterine artery Doppler at 11-14 weeks of gestation. Ultrasound Obstet Gynecol. 2001;18:583-586.

10. Figueras F, Gratacos E. Update on the diagnosis and classification of fetal growth restriction and proposal of a stage-based management protocol. Fetal Diagn Ther. 2014;36:86-98.

11. Valiño N, Giunta G, Gallo DM, Akolekar R, Nicolaides KH. Biophysical and biochemical markers at 30-34 weeks' gestation in the prediction of adverse perinatal outcome. Ultrasound Obstet Gynecol. 2016;47:194-202.

12. Valino N, Giunta G, Gallo DM, Akolekar R, Nicolaides KH. Biophysical and biochemical markers at 35-37 weeks' gestation in the prediction of adverse perinatal outcome. Ultrasound Obstet Gynecol. 2016:47:203-209.

13. Jaffa AJ, Weissman A, Har-Toov J, Shoham Z, Peyser RM. Flow velocity waveforms of the uterine artery in pregnancy: transvaginal versus transabdominal approach. Gynecol Obstet Invest. 1995:40:80-83.

14. Prefumo F, Bhide A, Sairam S, Penna L, Hollis B, Thilaganathan B. Effect of parity on second-trimester uterine artery Doppler flow velocity and waveforms. Ultrasound Obstet Gynecol. 2004;23:46-49.

15. Tayyar A, Guerra L, Wright A, Wright D, Nicolaides KH. Uterine artery pulsatility index in the three trimesters of pregnancy: effects of maternal characteristics and medical history. Ultrasound Obstet Gynecol. 2015;45:689-697.

16. O'Gorman N, Tampakoudis G, Wright A, Wright D, Nicolaides KH. Uterine artery pulsatility index at 12, 22, 32 and 36 weeks' gestation in screening for pre-eclampsia. Ultrasound Obstet Gynecol. 2016;47:565-572.

17. Oviedo-Cruz H, Cortés-Martínez MA. Presión arterial en embarazos normales de la Ciudad de México. Arch Cardiol Mex. 2021;91:289-298.

18. Royston $P$, Wright EM. How to construct "normal ranges" for fetal variables. Ultrasound Obstet Gynecol. 1998;11:30-38.

19. Katz MH. Multivariable analysis: a practical guide for clinicians and public health researchers. Inglaterra: Cambridge University Press; 2011.

20. Grant SW, Hickey GL, Head SJ. Statistical primer: multivariable regression considerations and pitfalls. Eur J Cardiothorac Surg. 2019;55:179-185

21. Carbillon L, Challier JC, Alouini S, Uzan M, Uzan S. Uteroplacental circulation development: Doppler assessment and clinical importance. Placenta. 2001;22:795-799.

22. Vinayagam D, Thilaganathan B, Stirrup O, Mantovani E, Khalil A. Maternal hemodynamics in normal pregnancy: reference ranges and role of maternal characteristics. Ultrasound Obstet Gynecol. 2018;51:665-671.

23. Aguree S, Gernand AD. Plasma volume expansion across healthy pregnancy: a systematic review and meta-analysis of longitudinal studies. BMC. 2019;19:508.

24. Oros D, Ruiz-Martínez S, Staines-Urias E, Conde-Agudelo A, Villar J, Fabre $\mathrm{E}$, et al. Reference ranges for Doppler indices of umbilical and fetal middle cerebral arteries and cerebroplacental ratio: systematic review. Ultrasound Obstet Gynecol. 2019;53:454-464.

25. Medina-Bastidas D, Guzmán-Huerta M, Borboa-Olivares H, Ruiz-Cruz C Parra-Hernández S, Flores-Pliego A, et al. Placental microarray profiling reveals common mRNA and IncRNA expression patterns in preeclampsia and intrauterine growth restriction. Int J Mol Sci. 2020;21:3597.

26. Rurangirwa AA, Gaillard R, Steegers EA, Hofman A, Jaddoe VW. Hemodynamic adaptations in different trimesters among nulliparous and multiparous pregnant women; the Generation R study. Am J Hypertens. 2012;25:892-899

27. Tay J, Masini G, McEniery CM, Giussani DA, Shaw CJ, Wilkinson IB, et al. Uterine and fetal placental Doppler indices are associated with maternal cardiovascular function. Am J Obstet Gynecol. 2019;220:96. e1-96.e8.

28. Morris EA, Hale SA, Badger GJ, Magness RR, Bernstein IM. Pregnancy induces persistent changes in vascular compliance in primiparous women. Am J Obstet Gynecol. 2015;212:633.e1-.e6.

29. Llurba E, Sánchez O, Domínguez C, Soro G, Goya M, Alijotas-Reig J, et al. Smoking during pregnancy: changes in mid-gestation angiogenic factors in women at risk of developing preeclampsia according to uterine artery Doppler findings. Hypertens Pregnancy. 2013;32:50-59. 